\title{
The incidence of skin lesions in contrast media- induced chemical hypersensitivity
}

\author{
ANDREI MIHAI IORDACHE ${ }^{*}$, ANCA OANA DOCEA ${ }^{2 *}$, ANA MARIA BUGA $^{3 *}$, RADU MITRUT $^{4 *}$, \\ DANA ALBULESCU ${ }^{5}$, OVIDIU ZLATIAN ${ }^{6}$, SIMONA IANOSI $^{7}$, GABRIEL IANOSI $^{8}$, \\ DANIELA NEAGOE ${ }^{9}$, MARIA SIFAKI $^{10}$, OTILIA CONSTANTINA ROGOVEANU ${ }^{11}$, \\ DACIANA ELENA BRANISTEANU ${ }^{12}$ and DANIELA CALINA ${ }^{1}$
}

\author{
Departments of ${ }^{1}$ Clinical Pharmacy, ${ }^{2}$ Toxicology, ${ }^{3}$ Biochemistry, ${ }^{4}$ Cardiology, ${ }^{5}$ Radiology, \\ ${ }^{6}$ Microbiology, ${ }^{7}$ Dermatology, ${ }^{8}$ Surgery and ${ }^{9}$ Internal Medicine, University of Medicine and Pharmacy of Craiova, \\ 200349 Craiova, Romania; ${ }^{10}$ Laboratory of Toxicology, Medical School, University of Crete, 71003 Heraklion, \\ Crete, Greece; ${ }^{11}$ Department of Physical Medicine and Rehabilitation, University of Medicine \\ and Pharmacy of Craiova, 200349 Craiova; ${ }^{12}$ Department of Dermatology, \\ University of Medicine and Pharmacy 'Gr. T. Popa', 700115 Iasi, Romania
}

Received August 03, 2018; Accepted October 08, 2018

DOI: $10.3892 /$ etm.2018.7056

\begin{abstract}
Contrast agents are used in radiology to increase the sensibility and specificity of radiological techniques. Some of these compounds have side effects that include organ toxicity (with kidney being the most affected organ) and hypersensitivity reactions. We performed multiple PubMed searches from January, 2008 to January, 2018 for studies regarding adverse reactions to compounds used as contrast agents in imagistic techniques. The initial research identified 929 records written in English. After further excluding 223 non-human studies, 292 articles that had irrelevant designs as reviews, meta-analysis, commentaries, editorials and case reports, 414 studies were selected for retrieval. After reading the abstracts, we excluded 363 studies as they had little relevance to the study. In total, 51 full-articles were assessed for eligible studies to be included. Finally, 20 articles were included in the analysis. In our systematic literature search the incidence of overall skin immediate reactions to iodinated contrast media (ICM) had an incidence between 1.15 and $0.12 \%$, depending on the cohort analyzed in the studies. The percentage of cutaneous manifestations
\end{abstract}

Correspondence to: Dr Anca Oana Docea, Department of Toxicology, University of Medicine and Pharmacy of Craiova, 2-4 Petru Rares Road, 200349 Craiova, Romania

E-mail: ancadocea@gmail.com

${ }^{*}$ Contributed equally

Key words: contrast agents, immediate hypersensitivity reactions, delayed hypersensitivity reactions, iodinated contrast agents, gadolinium-based contrast agents in the cohort that experienced immediate hypersensitivity reactions was between 33.33 and $87.7 \%$. The most frequent skin manifestations were urticaria, rashes, pruritus and limited facial edema. Non-iodinated contrast agents have a safer profile compared with ICM, the incidence of immediate adverse reactions being very low in gadolinium-based contrast agents and other agents used for contrast-enhanced ultrasound. The incidence of delayed reactions was between 10.1 and $0.03 \%$. In the studies analyzed by us the main adverse reactions due to delayed hypersensitivity phenomena were cutaneous manifestations that were present between 70.27 and $100 \%$ of the cases. Regarding the risk factors for developing immediate adverse reactions, being female was a predisposing factor accompanied by history of allergy and history of reactions to contrast media. An accurate anamnesis of the patients and a correctly conducted pretreatment can limit the incidence and the severity of the adverse reactions and also can avoid the life occurrence of life-threatening reactions.

\section{Introduction}

German physicist Walter Bradford was the first who discovered X-rays and their properties in November 1885. Their properties attracted medical researchers to use them in the medical field (1). To increase the sensibility and specificity of the radiological techniques, contrast media have been introduced into the medical practice. They are currently being used worldwide to increase the visibility of the investigated structures. Contrast agents were first used in the beginning of the 20th century, but with high incidence of toxicity and poor results. In the 1950's the use of contrast agents increased due to new formulations that became available. In the 1970's non-ionic dimeric contrast agents were developed and today they play a major role in diagnosis, being the most used contrast agents in daily radiology practice (2). 
Radiocontrast agents are typically iodine-, gadolinium- or barium-sulphate-based compounds and they can be administered orally or parenterally. Oral contrast agents are mostly used in the radiological diagnosis of the gastrointestinal tract. Contrast media for the bowel are classified as positive or negative, depending on whether the material is hyperattenuating or hypoattenuating relative to the walls of the gastrointestinal tract (3).

For the investigation of parenchymal organs and blood vessels, parenterally administered contrast media are used as iodinated contrast media (ICM) and gadolinium contrast media (4). Their administration is not totally safe, the adverse effects of the contrast media may be due to the type of administration, or to the type of agent used. The most common side effects after parenteral administration of contrast media are organ toxicity (with kidney being the most affected organ) (5) and hypersensitivity reactions (6).

The local side effect is represented by the contrast extravasation of the parenterally administrated agent, depending on the type of administration. As administration typically involves small volumes, it seldom leads to serious injuries. Non-communicative patients such as children or debilitated patients, multiple injections in the same vein, or friable vessels are considered to be risk factors (7).

The systemic side effects of the contrast media may occur early, usually in less than $20 \mathrm{~min}$, or late (over $20 \mathrm{~min}$ ), and the cause may be an anaphylactoid reaction or effects due to the osmolarity and chemotoxicity of the substance. The concentration, volume and rate of injection are also risk factors to be taken into consideration. Clinical reactions vary from minor, to intermediate, to severe and skin manifestations are the most frequent and sometimes the only manifestations that can be misinterpreted. The severe adverse reactions need an accurate diagnosis even if they are very rare because their evolution can be life-threatening $(8,9)$.

The aim of the study was to examine through a systematic analysis the studies published in the last 10 years regarding the incidence of immediate and delayed hypersensitivity reactions with skin manifestations, to identify the skin patterns that are characteristic in these types of reactions and to analyze the risk factors and comorbidities that can influence their appearance.

\section{Materials and methods}

Search for studies. We performed multiple PubMed searches using all possible combinations between the following keywords: Contrast agents or contrast media and immediate, non-immediate, late, delayed, allergic reactions, allergic effects, skin lesions, adverse effects, skin hypersensitivity and side effects. After analyzing the results only studies relevant to the subject were included in this systematic analysis. For a more accurate search we also checked the references of excluded studies.

Inclusion criteria were: Large observational studies that analyze the incidence of immediate and/or delayed hypersensitivity reactions to different class of contrast media and/or their association with the comorbidities, written in English language in the last 10 years, i.e., from January, 2008 till January, 2018.
Exclusion criteria were: Articles written in languages other than English, studies published before January 2008, non-human studies, studies published only as abstracts, as reviews, meta-analyses, commentaries, editorials, and case-reports but only after a careful check of those references. After reading the abstract and entire article we excluded from the systematic review the articles that did not clearly present the manifestations observed in the immediate and delayed reactions to the contrast media and studies that presented the incidence of other types of adverse reactions, as the purpose of this review was the skin manifestations in contrast media adverse reactions (Fig. 1).

Immediate hypersensitivity reactions to contrast media are defined as an allergic reaction that occurs within $30 \mathrm{~min}$ of contrast media administration. Delayed hypersensitivity reactions to contrast media are defined as reactions that appear between $1 \mathrm{~h}$ and 7 days after administration of the contrast agent (6).

\section{Results}

General. The initial research for the studies published in English between January 2008 and January 2018 yielded 929 articles. After excluding non-human studies and the reviews, meta-analyses, commentaries, editorials, and case-reports, 414 articles remained to be screened for their content by reading the abstract. In total, 51 full-articles were assessed for eligible studies to be included. Finally, 20 articles were included in the analysis. The selection procedure was carried out according to the inclusion and exclusion criteria and is presented in the PRISMA flow chart (Fig. 1). We identified 11 studies that described immediate skin reactions to ICM, 9 studies that described immediate skin reactions to other contrast media, 6 studies that described delayed skin adverse reactions to ICM, and 3 studies that described delayed skin adverse reactions to other contrast media. Regarding the risk factors associated with the incidence of immediate and delayed allergic reactions from the studies included in the systematic review, 7 studies analyzed the risk factors associated with immediate allergic reactions to ICM and 4 studies analyzed the risk factors associated with immediate allergic reactions to other contrast agents. No study analyzed the risk factors associated with delayed allergic reactions.

Incidence of immediate skin adverse reactions to ICM. The epidemiological studies published in the last 10 years that evaluate the incidence of immediate adverse reactions to ICM with skin manifestations identify a prevalence of immediate hypersensitivity reactions between 0.16 and $2.24 \%$, from which the percentage of skin manifestation was between 33.33 and $87.7 \%$ (Table I) (9-19). Severe hypersensitivity reactions were between 0.010 and $0.024 \%$, with anaphylaxis being the most frequent reaction (Table I) $(9,10,18)$. Regarding the skin patterns observed in these cases, the most frequent were urticaria (between 30.77 and $83.78 \%$ of the cases with skin manifestations) $(10,13,15,17-19)$, rash $(38.46-85.3 \%)(9,10,13,19)$, itching sensation/pruritus (12.82-100\%) (10,13-15,17-19), oedema $(6.25-17.3 \%)(12,18,19)$, erythemas $(36.54-100 \%)(12,14,17-19)$, angioedema $(8 \%-13.51 \%)(15,17)$, and angioneurotic oedema (3.84\%) (18) (Fig. 2). 
PRISMA flow diagram

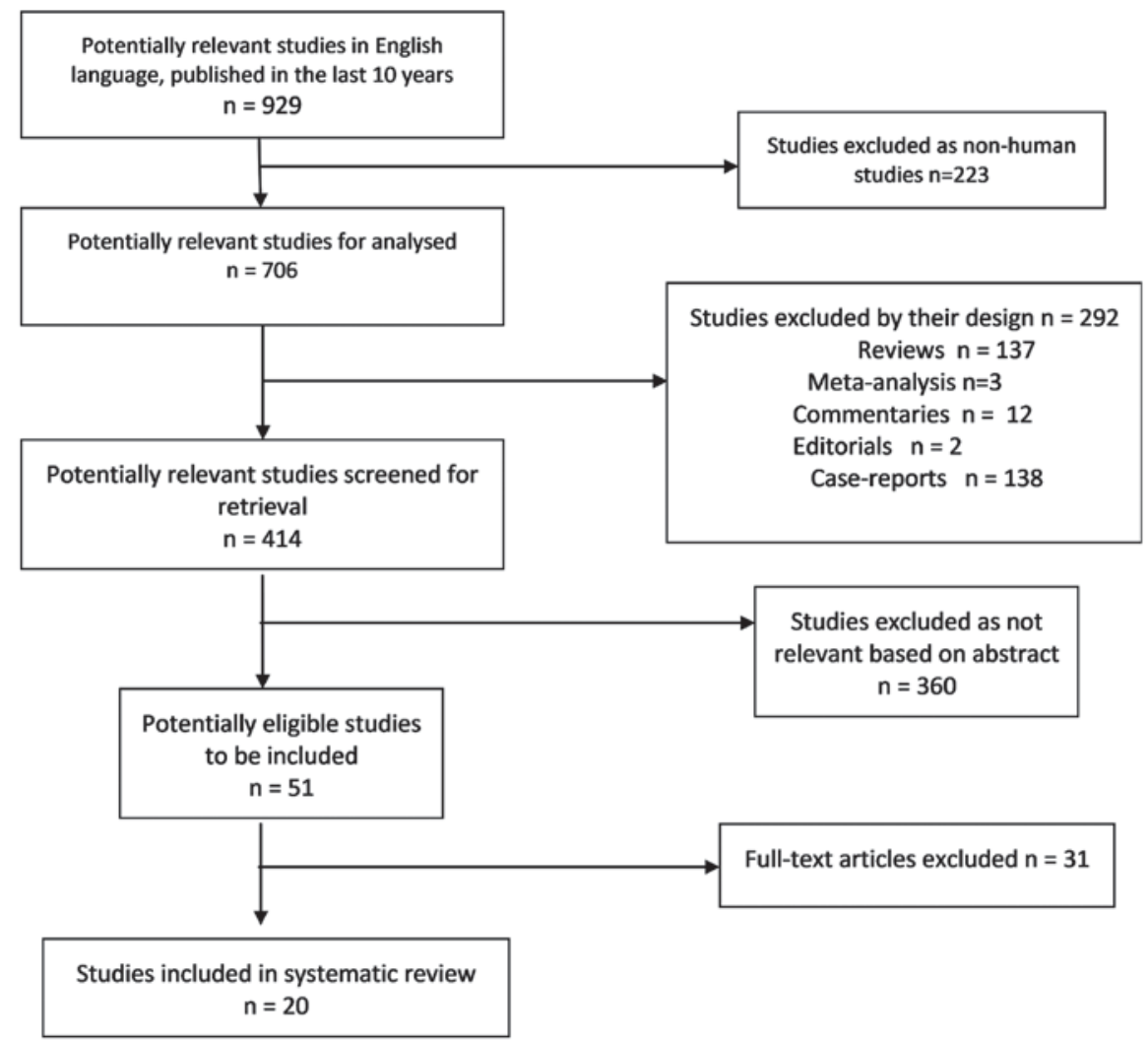

Figure 1. PRISMA flow chart of study.

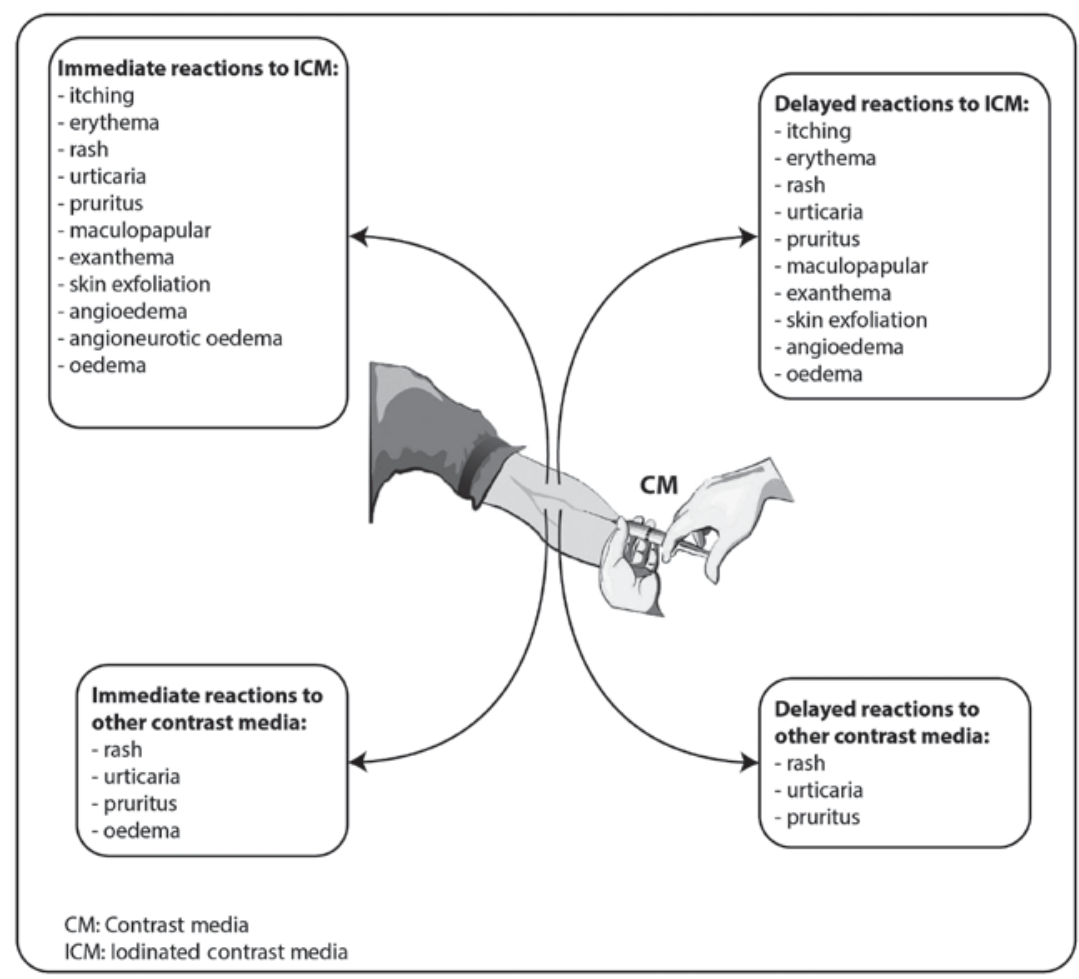

Figure 2. Main skin patterns observed in immediate and delayed hypersensitivity reactions to contrast media.

Incidence of immediate skin adverse reactions to other contrast media. In Table II are presented the studies published in the last 10 years that evaluate the incidence of immediate adverse reactions to other contrast media except ICM with 


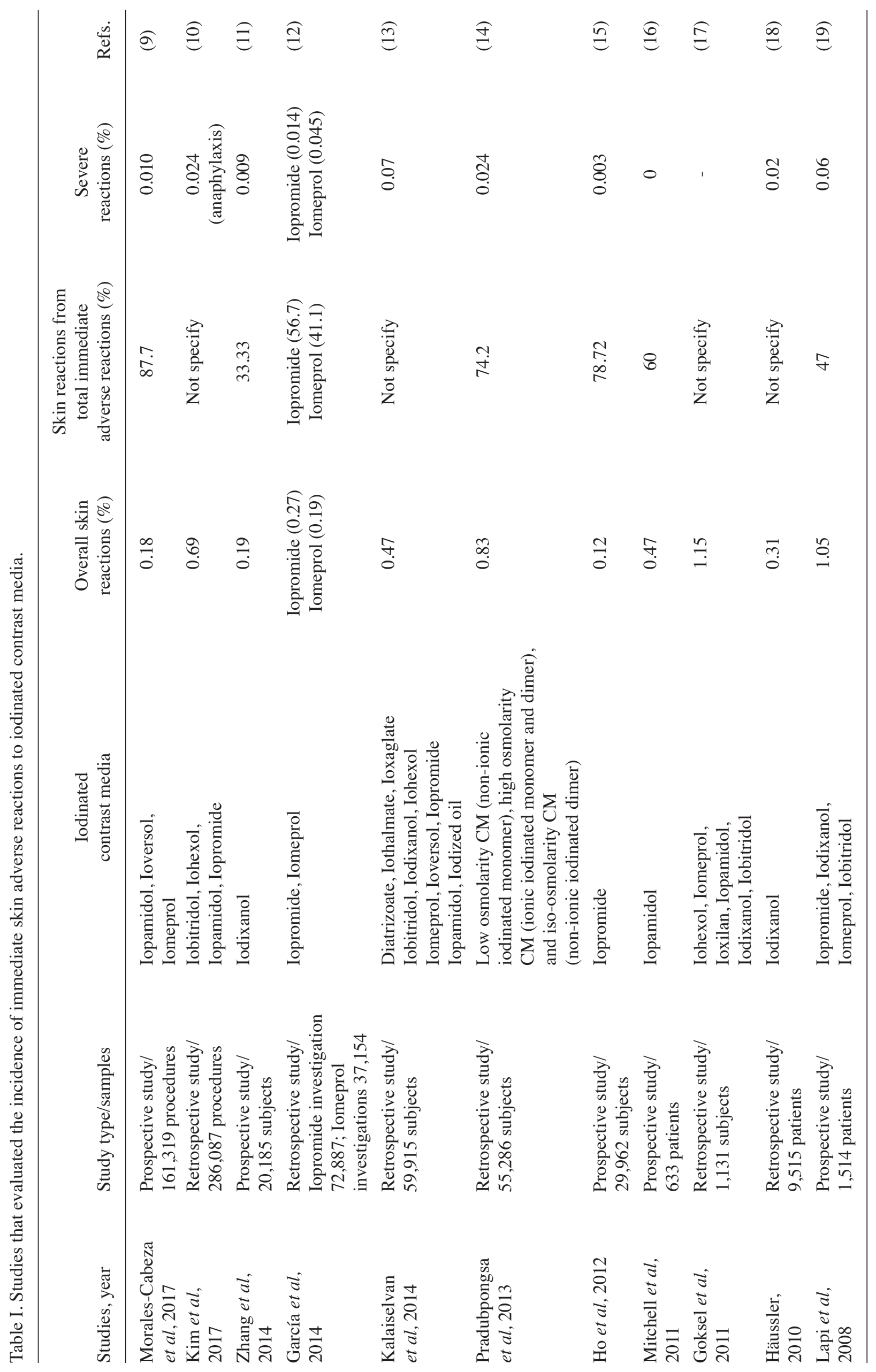




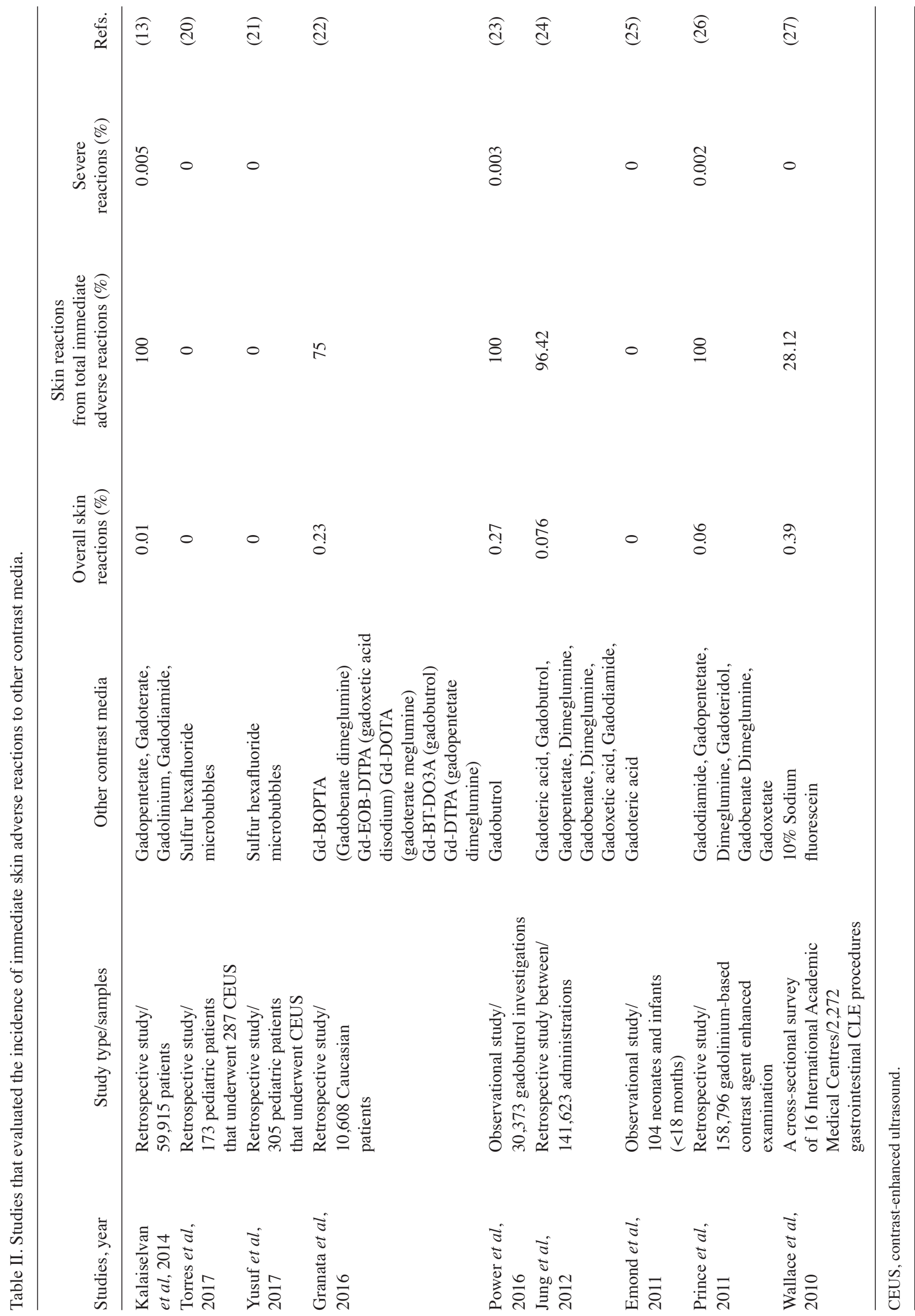


skin manifestations. We identified 6 studies that evaluated the immediate reactions to gadolinium-based contrast agents, 2 studies that evaluated the immediate reactions to sulfur hexafluoride microbubbles, a contrast agent used for contrast-enhanced ultrasound and 1 study that evaluated the immediate hypersensitivity reactions to sodium fluorescein.

Two studies investigated the immediate adverse reactions to sulfur hexafluoride microbubbles in pediatric patients. No immediate hypersensitivity effects were evident after the administration $(20,21)$.

Regarding the gadolinium contrast media the incidence of immediate hypersensitivity reactions was between 0.01 and $0.3 \%$. The skin manifestations were observed in $75-100 \%$ of these reactions $(13,22-27)$. The most frequent skin manifestations associated with hypersensitivity reactions to gadolinium contrast agents were urticaria $(63-91.1 \%)(13,22-24)$, rash $(20.4 \%)(13,22,23)$, pruritus (22.2\%) (23), limited facial edema (6.17\%) (23). Regarding the life-threatening severe hypersensitivity reactions only Kalaiselvan et al reported one case manifested by laryngospasm (13) (Fig. 2).

Wallace et al evaluated the safety of fluorescein, a contrast agent utilized for confocal laser endomicroscopy. They determined an incidence of $1.4 \%$ of immediate side effects from which $28.12 \%$ had skin manifestations. The skin patterns observed were injection side erythema 88 and rash 12\% (from the cases with skin effects) (27).

Incidence of delayed skin adverse reactions to ICM. The delayed hypersensitivity reactions were also reported in the studies published in the last 10 years that investigated the safety of ICM (Table III). A prevalence of 0.42 and $14.3 \%$ was observed, from which the incidence of skin manifestations were between 43.05 and $100 \%(11,14,17-19,28)$. The most frequent skin pattern was angioedema (between 11.1 and $43.7 \%)(13,16)$, itching/pruritus (18.7-55.6\%) $(10,13,16-18$, $27)$ and maculopapular exanthema $(33.3-37.5 \%)(14,17)$. Other reactions with low incidence were erythema, rash, urticarial, and oedema (11,14,17-19,28) (Fig. 2).

In a study by Häussler 3 cases (0.03\%) of severe delayed hypersensitivity reactions were reported: i) One 59-year-old female that experienced hypersensitivity, pruritus, urticarial, increased blood pressure, vomiting, swelling of the face, eyelid edema, facial edema, diarrhea and tachycardia; ii) one 53-year-old female that presented hypersensitivity, swelling of the face, erythema, and skin irritation and; iii) one 79-year-old male that presented hypersensitivity, rash, pruritus, dermatitis, and erythema. All 3 cases needed hospitalization (18).

Incidence of delayed skin adverse reactions to other contrast media. Delayed skin reactions to other contrast media except ICM were investigated in only 3 studies published in the last 10 years (Table IV). Two studies evaluated the safety of sulfur hexafluoride microbubbles in pediatric population and reported no delayed adverse effects $(20,21)$. Power et al reported an incidence of $0.05 \%$ delayed sensitivity reactions to gadolinium-based contrast agents with skin lesions. The symptoms observed were urticaria (66\%), rash (33\%) and pruritus (6.6\%). Delayed reactions occurred on the same day in $46 \%$ of cases, on the following day in $20 \%$ of cases and in $33 \%$ of cases the moment of manifestation was uncertain (23) (Fig. 2).

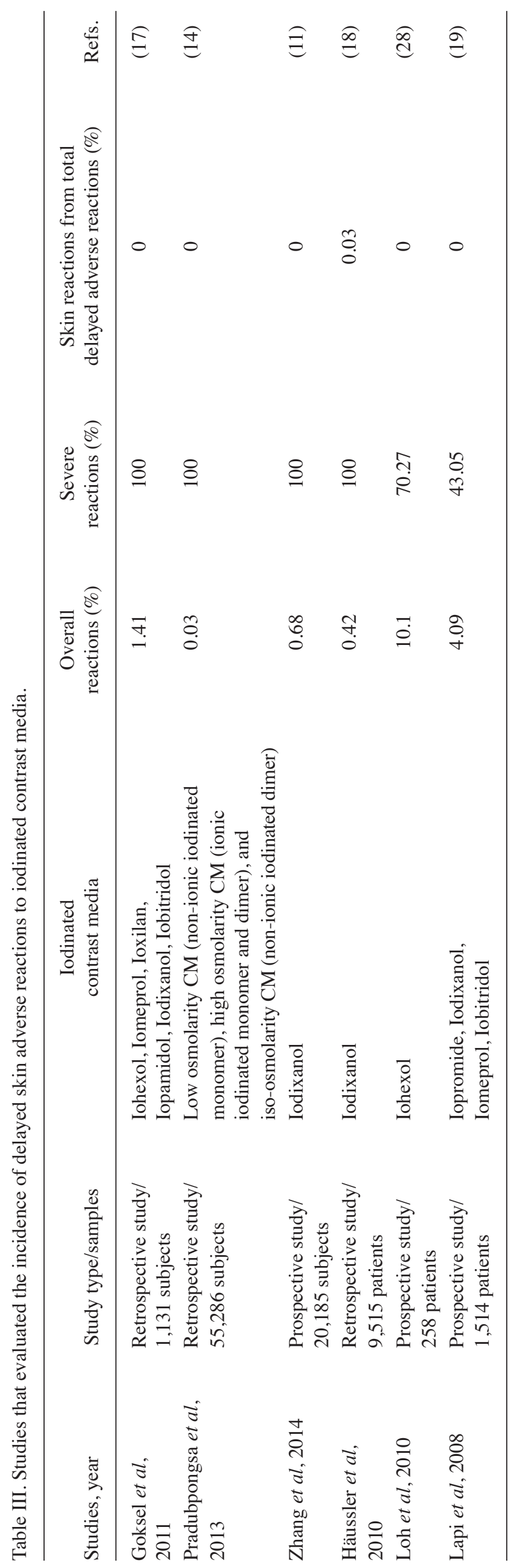


Table IV. Studies that evaluated the incidence of delayed skin adverse reactions to other contrast media.

\begin{tabular}{|c|c|c|c|c|c|c|}
\hline Studies, year & $\begin{array}{c}\text { Study type/ } \\
\text { samples }\end{array}$ & $\begin{array}{l}\text { Iodinated } \\
\text { contrast } \\
\text { media }\end{array}$ & $\begin{array}{l}\text { Overall } \\
\text { reactions } \\
(\%)\end{array}$ & $\begin{array}{l}\text { Skin reactions from } \\
\text { total delayed adverse } \\
\text { reactions }(\%)\end{array}$ & $\begin{array}{l}\text { Severe } \\
\text { reactions } \\
(\%)\end{array}$ & Refs. \\
\hline $\begin{array}{l}\text { Torres et al, } \\
2017\end{array}$ & $\begin{array}{l}\text { Retrospective study/ } \\
173 \text { pediatric patients } \\
\text { that underwent } \\
287 \text { CEUS }\end{array}$ & $\begin{array}{l}\text { Sulfur } \\
\text { hexafluoride } \\
\text { microbubbles }\end{array}$ & 0 & 0 & 0 & (20) \\
\hline $\begin{array}{l}\text { Yusuf et al, } \\
2017\end{array}$ & $\begin{array}{l}\text { Retrospective study/ } \\
305 \text { pediatric patients } \\
\text { that underwent CEUS }\end{array}$ & $\begin{array}{l}\text { Sulfur } \\
\text { hexafluoride } \\
\text { microbubbles }\end{array}$ & 0 & 0 & 0 & (21) \\
\hline $\begin{array}{l}\text { Power et al, } \\
2016\end{array}$ & $\begin{array}{l}\text { Observational study/ } \\
30,373 \text { gadobutrol } \\
\text { investigations }\end{array}$ & Gadobutrol & 0.05 & 100 & 0 & $(23)$ \\
\hline
\end{tabular}

CEUS, contrast-enhanced ultrasound.

Risk factors associated with immediate allergic reactions to $I C M$. Several risk factors were associated with the appearance of immediate hypersensitivity reactions to ICM. We identified 7 studies there of (Table V). The female sex was associated with increased risk for immediate allergic reactions to ICM between 51.44 and $65.95 \%(9,10,14,15,17,18)$. Other risk factors identified included history of previous reactions to ICM $(1.2-11.6 \%)(9,12-15,17)$, atopy $(14.3 \%)(8)$, asthma $(2.1-12.7 \%)$ $(9,14,15,17)$, drug allergy $(3.6-25 \%)(9,14,15,17)$, and allergic rhinitis $(1.5-4 \%)(14,15,17)$.

The study of Morales-Cabeza et al associated the immediate allergic reactions to concomitant treatment with $\beta$-blockers $(7.9 \%)$ or angiotensin-converting enzyme inhibitors (ACEI) (13.2\%) (9).

Risk factors associated with immediate allergic reactions to other contrast media. Being female was associated with gadolinium-based contrast agents and immediate adverse reactions in $65.2-81.25 \%$ of cases $(22-24,26)$. Immediate adverse reactions to other contrast media were associated with previous reaction to gadolinium contrast media $(7.31-8.5 \%)(23,26)$, asthma $(2.9-11 \%)(23,24,26)$, drug hypersensitivity (2\%) (24), allergic rhinitis (2.9\%) (24), and chronic urticaria (2\%) (24) (Table VI).

\section{Discussion}

In our systematic literature search the incidence of overall skin immediate reactions to ICM had an incidence between 1.15 and $0.12 \%$, depending on the cohort analyzed in the studies. Goksel et al (17) analyzed only a cohort of 1,131 patients and observed an incidence of $1.15 \%$ of immediate reactions with skin manifestations in comparison with Ho et al (15) who analyzed a cohort of 29,962 patients and determined an incidence of $0.12 \%$. The percentage of cutaneous manifestations in the cohort that experienced immediate hypersensitivity reactions was between 33.33 and $87.7 \%$, with most of the studies reporting percentages above $50 \%$. The most frequent symptoms observed were urticaria, rash, itching and erythemas. Severe immediate adverse reactions, anaphylactic manifestations being the most frequent, were also identified. Those results are in accordance with the older studies, which reported a prevalence of 3.8 and $12.7 \%$ of mild immediate $\mathrm{CM}$ reactions after using an ionic-iodinated $\mathrm{CM}$ and between 0.7 and 3.1\% for non-ionic-iodinated CM (29-32). Most of the studies discussed in the present systematic analysis reported results after exposure to non-ionic ICM.

The mechanism of CM-induced allergic reaction is unclear and multifactorial and it is considered that more mechanisms are involved. The main mechanism considered is type-I hypersensitivity mechanism. Other proposed mechanisms are associated with histamine release from mast cells and basophils or activation of the complement system. Studies have shown that osmolarity or chemical structure of ICM can act directly on the cells and determine direct membrane effects that lead to these types of reactions (33). Previous findings showed that some ICM activate one of the true hypersensitivity pathways. The lower rate of adverse reactions to gadolinium-based contrast media is probably due to limited data available (34). Therefore, most immediate adverse reactions to contrast media probably occur through different pathways, supported by high level of histamine observed in the serum of patients with this type of reactions (33).

Non-iodinated contrast agents have a safer profile compared with ICM, the incidence of immediate adverse reactions being very low in gadolinium-based contrast agents and other agents used for contrast-enhanced ultrasound (CEUS). Previous studies showed that gadolinium-based contrast agents determine mild, moderate and severe immediate adverse reactions with an incidence of 0.07 and 3.1\% (35-37), which is in accordance with our findings. The incidence of immediate adverse reactions was identified as 0.01 and $1.3 \%$, from which the skin manifestations were presented in 75 and $100 \%$ of cases. The most frequent skin manifestations were urticaria, rashes, pruritus and limited facial edema. Regarding other contrast agents, the ones used for CEUS are considered a safer alternative, especially in pediatric population where CT and MRI investigations present numerous disadvantages 


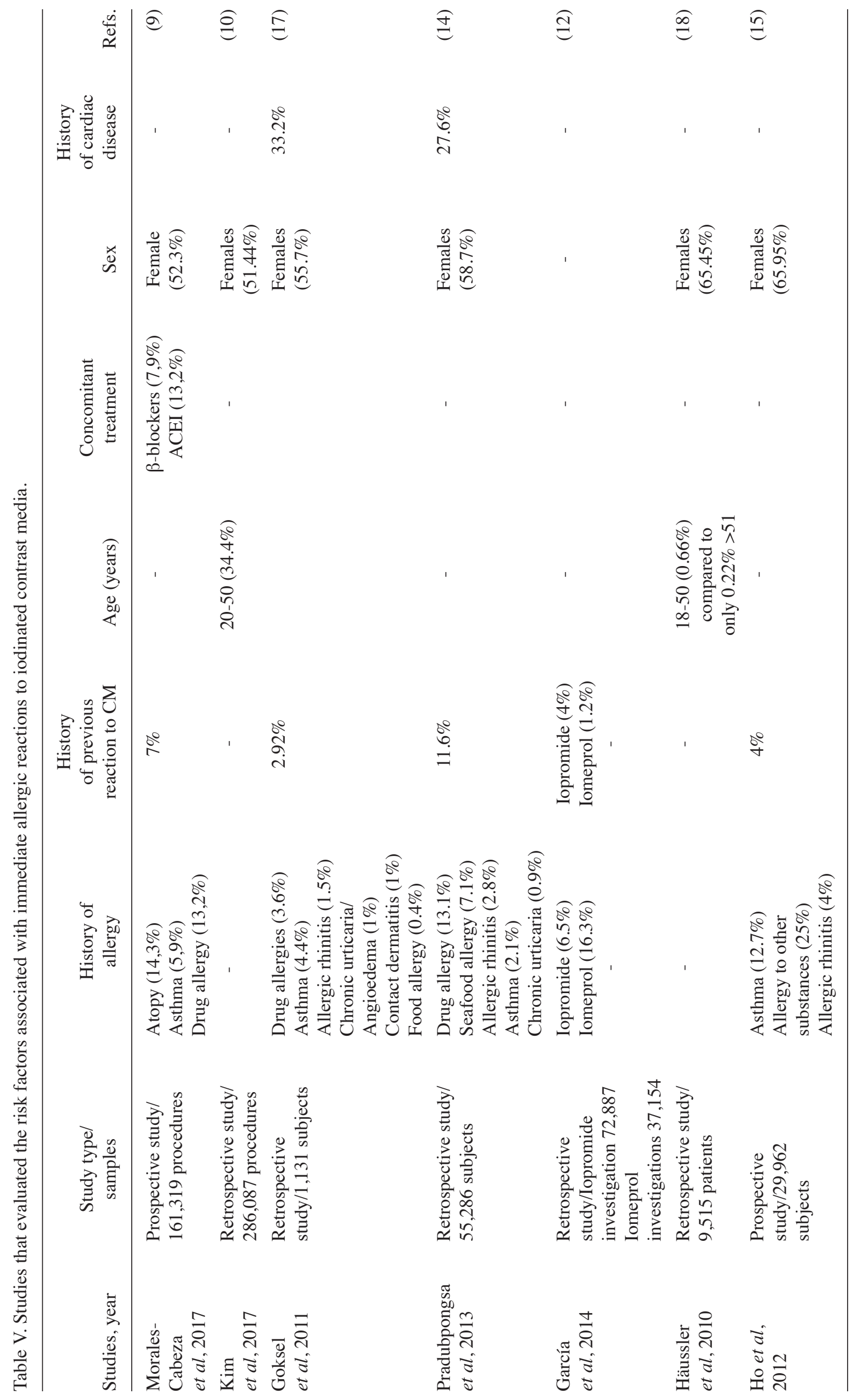




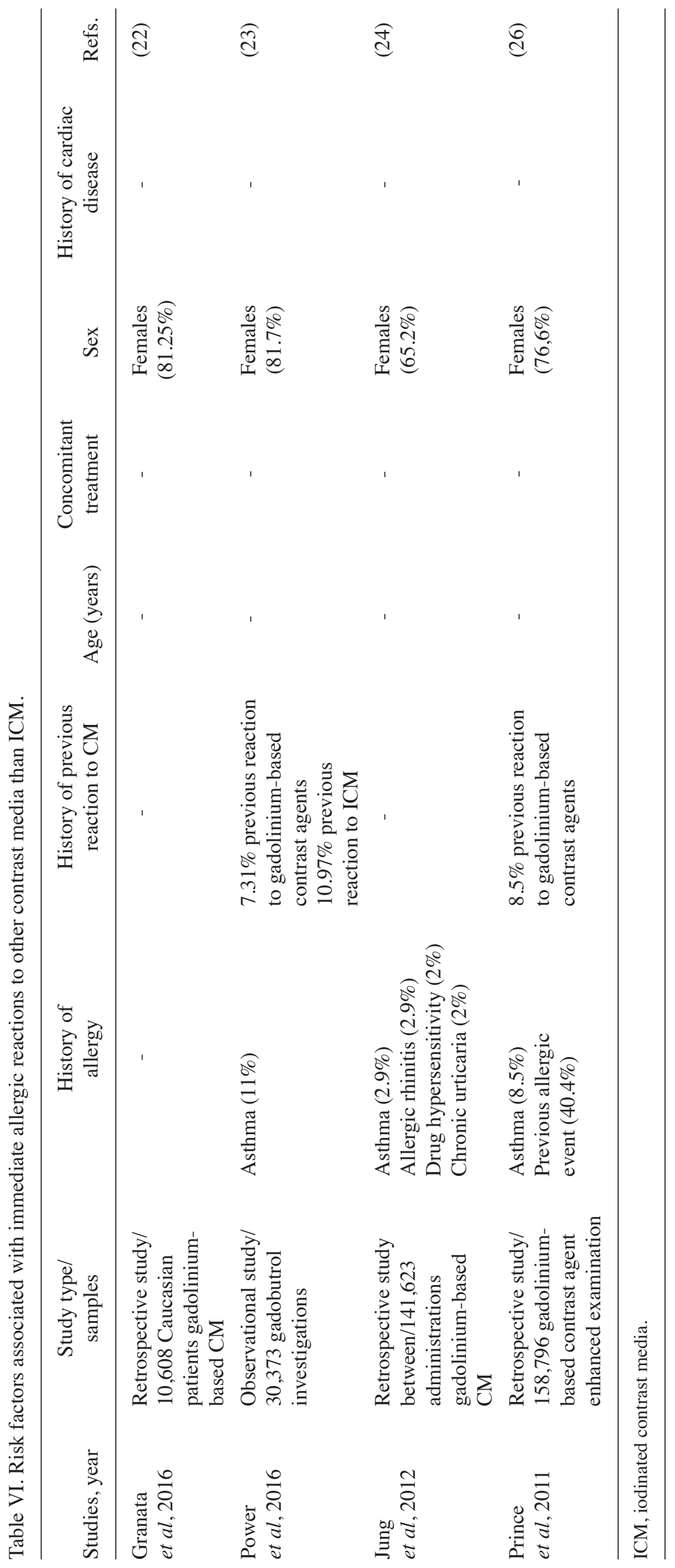


with regard to sedation or general anesthesia. The incidence of immediate adverse reactions to CEUS agents was very low, around $0.0086 \%$ (38). In our systematic analysis, we identified 2 studies that reported no immediate adverse reaction to sulfur hexafluoride microbubbles used as CM for CEUS in pediatric population.

In the studies analyzed in our systematic analysis the incidence of delayed reactions to ICM was between 10.1 and $0.03 \%$. The higher incidence observed can also be determined because of the low cohort investigated of only 258 patients compared with the study where the lowest incidence was observed, which analyzed 55,286 subjects. Older studies showed an incidence of delayed reactions to ICM of 2 and 5\% (39-42). In the studies we analyzed the main adverse reactions due to delayed hypersensitivity phenomena were cutaneous manifestations that were present between 70.27 and $100 \%$ of the cases analyzed. The skin manifestations in the mild and moderate reactions were rash, urticaria, pruritus or erythema and angioedema. The severe reactions are very rare but life threatening, being a significant problem for both the patients and physicians involved. Between the skin patterns observed in the severe delayed reactions there were reported multiform erythema (43), cutaneous vasculitis (44), fixed drug eruption (45), Stevens-Johnson syndrome (46), and toxic epidermal necrolysis (47). We identified only one study that reported 3 cases of severe delayed reactions (0.03\%) from 9,515 patients exposed to contrast agents (18). The skin manifestations observed in these patients were pruritus, urticarial, swelling of the face, eyelid edema, facial edema, rash and erythema (18).

The mechanism of appearance of these delayed reactions is mediated via T cells. This mechanism is supported by the studies that showed the presence of dermal infiltrates of $\mathrm{T}$ cells in affected skin and at positive skin test sites, the reappearance of the eruption after provocation testing and by the ability of $\mathrm{CM}$ to stimulate the proliferation of peripheral $\mathrm{T}$ cells from patients with CM-induced skin eruptions (48).

The delayed sensitivity reactions to other contrast media except ICM are rarely reported, also because these products are on the market for a short period (49). We identified only one study that reported an incidence of $0.05 \%$ delayed sensitivity reactions and the skin manifestations observed were urticarial, rash and pruritus (23). No severe reactions were reported (23).

The hypersensitivity reactions to ICM are associated with certain associated factors that may be risk factors and a correct investigation of the patient before the use of a certain contrast agent can be beneficial for decreasing the risk of its manifestation. History of allergy, history of previous reactions to CM, age less than 50 years, female sex, history of cardiac disease, and concomitant treatment with other drugs such as $\beta$-blockers or ACEI were identified as risk factors, which is in accordance with older studies $(50,51)$. History of asthma was associated with severe reactions, thus in these patients other alternatives could be suggested.

Regarding the risk factors for developing immediate adverse reactions to gadolinium-based CM, the studies showed a similar pattern as those that appear after exposure to ICM, female gender being a predisposing factor, a percentage between 65.2 and $81.7 \%$ from those that developed immediate hypersensitivity reactions to gadolinium-based contrast agents were women, findings that are in concordance with the older literature $(26,52)$. These differences are not well explained, but animal studies suggested that specific sex hormones may be involved in the increased incidence in females (53). History of allergy and history of previous reaction to $\mathrm{CM}$ is an important determinant factor. In the studies analyzed, age and concomitant treatment were not found to be risk factors, even if other studies reported them as such (54).

In conclusion, contrast agents are extensively used at present for a large range of imagistic investigations and their utilization is not totally safe. Irrespective of the class of contrast agents to which they belong, immediate or delayed adverse reactions could appear, ICM presenting a higher incidence compared with new agents as gadolinium-based contrast agents or others utilized for CEUS. Skin manifestations are the most frequent manifestations in all the type of allergic reactions and a precise diagnosis can be very helpful in practice. Several risk factors were associated with the appearance of immediate hypersensitivity reactions to contrast media. An accurate anamnesis of the patients and a correctly conducted pretreatment can limit the incidence and the severity of the adverse reactions and also can avoid the occurrence of life threatening reactions.

\section{Acknowledgements}

This study is part of the PhD theses of Andrei Mihai Iordache from the University of Medicine and Pharmacy of Craiova, Craiova, Romania.

\section{Funding}

No funding was received.

\section{Availability of data and materials}

The datasets used and/or analyzed during the present study are available from the corresponding author on reasonable request.

\section{Authors' contributions}

AMI, AOD, AMB, RM and DC contributed to the interpretation of study, performed the systematic search of the literature, writing the manuscript and revising it critically for important intellectual content. DA, OZ, SI, GI, DN, MS, OCR and DEB are responsible for the acquisition, analysis and interpretation of the data. All authors read and approved the final manuscript.

\section{Ethics approval and consent to participate}

Not applicable.

\section{Patient consent for publication}

Not applicable.

\section{Competing interests}

The authors declare that they have no competing interests. 


\section{References}

1. Filler A: The History, development and impact of computed imaging in neurological diagnosis and neurosurgery: CT, MRI, and DTI. Nat Preced 7: 1-76, 2009. https://doi.org/10.1038/ npre.2009.3267.

2. Singh $J$ and Daftary A: Iodinated contrast media and their adverse reactions. J Nucl Med Technol 36: 69-77, 2008.

3. Hasegawa $\mathrm{M}$ and Gomi T: Radiological contrast agents and radiopharmaceuticals. In: Side Effects of Drugs Annual. Ray SD (ed). Vol 37. 1st edition. Elsevier, Amsterdam, pp583-593, 2015.

4. Iyer RS, Schopp JG, Swanson JO, Thapa MM and Phillips GS: Safety essentials: Acute reactions to iodinated contrast media. J Can Assoc Radiol 64: 193-199, 2013.

5. Mamoulakis C, Tsarouhas K, Fragkiadoulaki I, Heretis I, Wilks MF, Spandidos DA, Tsitsimpikou C and Tsatsakis A: Contrast-induced nephropathy: Basic concepts, pathophysiological implications and prevention strategies. Pharmacol Ther 180: 99-112, 2017.

6. Sodagari F, Mozaffary A, Wood CG III, Schmitz B, Miller FH and Yaghmai V: Reactions to both nonionic iodinated and gadolinium-based contrast media: Incidence and clinical characteristics. AJR Am J Roentgenol 210: 715-719, 2018.

7. Nicola R, Shaqdan KW, Aran S, Prabhakar AM, Singh AK and Abujudeh $\mathrm{HH}$ : Contrast media extravasation of computed tomography and magnetic resonance imaging: Management guidelines for the radiologist. Curr Probl Diagn Radiol 45 161-164, 2016

8. Fok JS and Smith WB: Hypersensitivity reactions to gadolinium-based contrast agents. Curr Opin Allergy Clin Immunol 17: 241-246, 2017.

9. Morales-Cabeza C, Roa-Medellin D, Torrado I, De Barrio M, Fernández-Álvarez C, Montes-Aceñero JF, De La Riva I, Prieto-García A: Immediate reactions to iodinated contrast media. Ann Allergy Asthma Immunol 119: 553-557, 2017.

10. Kim SR, Lee JH, Park KH,Park HJ and Park JW: Varied incidence of immediate adverse reactions to low-osmolar non-ionic iodide radiocontrast media used in computed tomography. Clin Exp Allergy 47: 106-112, 2017.

11. Zhang BC, Hou L, Lv B and Xu YW: Post-marketing surveillance study with iodixanol in 20185 Chinese patients from routine clinical practices. Br J Radiol 87: 20130325, 2014.

12. García M, Aguirre U, Martinez A, Ruiz B, Lertxundi U and Aguirre C: Acute adverse reactions to iopromide vs iomeprol: A retrospective analysis of spontaneous reporting from a radiology department. Br J Radiol 87: 20130511, 2014.

13. Kalaiselvan V, Sharma S and Singh GN: Adverse reactions to contrast media: An analysis of spontaneous reports in the database of the pharmacovigilance programme of India. Drug Saf 37: 703-710, 2014

14. Pradubpongsa P, Dhana N, Jongjarearnprasert K, Janpanich S and Thongngarm T: Adverse reactions to iodinated contrast media: Prevalence, risk factors and outcome-the results of a 3-year period. Asian Pac J Allergy Immunol 31: 299-306, 2013

15. Ho J, Kingston RJ, Young N, Katelaris CH and Sindhusake D: Immediate hypersensitivity reactions to IV non-ionic iodinated contrast in computed tomography. Asia Pac Allergy 2: 242-247, 2012.

16. Mitchell AM, Jones AE, Tumlin JA and Kline JA: Immediate complications of intravenous contrast for computed tomography imaging in the outpatient setting are rare. Acad Emerg Med 18 : 1005-1009, 2011

17. Goksel O, Aydın O, Atasoy C, Akyar S, Demirel YS, Misirligil Z and Bavbek S: Hypersensitivity reactions to contrast media: Prevalence, risk factors and the role of skin tests in diagnosis - a cross-sectional survey. Int Arch Allergy Immunol 155: 297-305, 2011.

18. Häussler MD: Safety and patient comfort with iodixanol: A postmarketing surveillance study in 9515 patients undergoing diagnostic CT examinations. Acta Radiol 51: 924-933, 2010.

19. Lapi F, Cecchi E, Pedone C, Attanasio F, Banchelli G, Vannacci A, Di Pirro M, Moschini M, Berni V, Matucci R, et al: Safety aspects of iodinated contrast media related to their physicochemical properties: A pharmacoepidemiology study in two Tuscany hospitals. Eur J Clin Pharmacol 64: 723-737, 2008

20. Torres A, Koskinen SK, Gjertsen H and Fischler B Contrast-enhanced ultrasound using sulfur hexafluoride is safe in the pediatric setting. Acta Radiol 58: 1395-1399, 2017.
21. Yusuf GT, Sellars ME, Deganello A, Cosgrove DO and Sidhu PS: Retrospective analysis of the safety and cost implications of pediatric contrast-enhanced ultrasound at a single center. AJR Am J Roentgenol 208: 446-452, 2017.

22. Granata V, Cascella M, Fusco R, dell'Aprovitola N, Catalano O, Filice S, Schiavone V, Izzo F, Cuomo A and Petrillo A: Immediate adverse reactions to gadolinium-based MR contrast media: A retrospective analysis on 10,608 examinations. BioMed Res Int 2016: 3918292, 2016. doi.org/10.1155/2016/3918292.

23. Power S, Talbot N, Kucharczyk W and Mandell DM Allergic-like reactions to the MR imaging contrast agent gadobutrol: A prospective study of 32991 consecutive injections. Radiology 281: 72-77, 2016.

24. Jung JW, Kang HR, Kim MH, Lee W, Min KU, Han MH and Cho SH: Immediate hypersensitivity reaction to gadolinium-based MR contrast media. Radiology 264: 414-422, 2012.

25. Emond S and Brunelle F: Gd-DOTA administration at MRI in children younger than 18 months of age: Immediate adverse reactions. Pediatr Radiol 41: 1401-1406, 2011.

26. Prince MR, Zhang H, Zou Z, Staron RB and Brill PW: Incidence of immediate gadolinium contrast media reactions. AJR Am J Roentgenol 196: 138-143, 2011.

27. Wallace MB, Meining A, Canto MI, Fockens P, Miehlke S, Roesch T, Lightdale CJ, Pohl H, Carr-Locke D, Löhr M, et al: The safety of intravenous fluorescein for confocal laser endomicroscopy in the gastrointestinal tract. Aliment Pharmacol Ther 31: 548-552, 2010.

28. Loh S, Bagheri S, Katzberg RW, Fung MA and Li CS: Delayed adverse reaction to contrast-enhanced CT: A prospective single-center study comparison to control group without enhancement. Radiology 255: 764-771, 2010.

29. Katayama H, Yamaguchi K, Kozuka T, Takashima T, Seez P and Matsuura K: Adverse reactions to ionic and nonionic contrast media. A report from the Japanese committee on the safety of contrast media. Radiology 175: 621-628, 1990.

30. Wolf GL, Arenson RL and Cross AP: A prospective trial of ionic vs nonionic contrast agents in routine clinical practice: Comparison of adverse effects. AJR Am J Roentgenol 152: 939-944, 1989

31. Palmer FJ: The RACR survey of intravenous contrast media reactions. Final report. Australas Radiol 32: 426-428, 1988.

32. Caro JJ, Trindade E and McGregor M: The risks of death and of severe nonfatal reactions with high- vs low-osmolality contrast media: A meta-analysis. AJR Am J Roentgenol 156: 825-832, 1991.

33. Idée JM, Pinès E, Prigent $P$ and Corot $C$ : Allergy-like reactions to iodinated contrast agents. A critical analysis. Fundam Clin Pharmacol 19: 263-281,2005.

34. Boyd B,Zamora CA and Castillo M: Managing adverse reactions to contrast agents. Magn Reson Imaging Clin N Am 25: 737-742, 2017.

35. Kirchin MA and Runge VM: Contrast agents for magnetic resonance imaging: Safety update. Top Magn Reson Imaging 14: 426-435, 2003

36. Ersoy $\mathrm{H}$ and Rybicki FJ: Biochemical safety profiles of gadolinium-based extracellular contrast agents and nephrogenic systemic fibrosis. J Magn Reson Imaging 26: 1190-1197, 2007.

37. Nelson KL, Gifford LM, Lauber-Huber C, Gross CA and Lasser TA: Clinical safety of gadopentetate dimeglumine. Radiology 196: 439-443, 1995.

38. Piscaglia $\mathrm{F}$ and Bolondi L; Italian society for ultrasound in medicine and biology (SIUMB) study group on ultrasound contrast agents: The safety of Sonovue in abdominal applications: Retrospective analysis of 23188 investigations. Ultrasound Med Biol 32: 1369-1375, 2006.

39. Yasuda $\mathrm{R}$ and Munechika H: Delayed adverse reactions to nonionic monomeric contrast-enhanced media. Invest Radiol 33: $1-5,1998$.

40. Munechika H, Hiramatsu Y, Kudo S, Sugimura K, Hamada C, Yamaguchi $\mathrm{K}$ and Katayama $\mathrm{H}$ : A prospective survey of delayed adverse reactions to iohexol in urography and computed tomography. Eur Radiol 13: 185-194, 2003.

41. Rydberg J, Charles J and Aspelin P: Frequency of late allergy-like adverse reactions following injection of intravascular non-ionic contrast media. A retrospective study comparing a non-ionic monomeric contrast medium with a non-ionic dimeric contrast medium. Acta Radiol 39: 219-222, 1998.

42. Panto PN and Davies P: Delayed reactions to urographic contrast media. Br J Radiol 59: 41-44, 1986. 
43. Akiyama M, Nakada T, Sueki H, Fujisawa R and Iijima M: Drug eruption caused by nonionic iodinated $\mathrm{X}$-ray contrast media. Acad Radiol 5: 159-161, 1998.

44. Reynolds NJ, Wallington TB and Burton JL: Hydralazine predisposes to acute cutaneous vasculitis following urography with iopamidol. Br J Dermatol 129: 82-85, 1993.

45. Good AE, Novak E and Sonda LP III: Fixed eruption and fever after urography. South Med J 73: 948-949, 1980.

46. Savill JS, Barrie R, Ghosh S, Muhlemann M, Dawson P and Pusey CD: Fatal Stevens-Johnson syndrome following urography with iopamidol in systemic lupus erythematosus. J Postgrad Med 64: 392-394, 1988.

47. Rosado A, Canto G, Veleiro B and Rodríguez J: Toxic epidermal necrolysis after repeated injections of iohexol. AJR Am J Roentgenol 176: 262-263, 2001.

48. Kanny G, Pichler W, Morisset M, Franck P, Marie B, Kohler C, Renaudin JM, Beaudouin E, Laudy JS and Moneret-Vautrin DA: T cell-mediated reactions to iodinated contrast media: Evaluation by skin and lymphocyte activation tests. J Allergy Clin Immunol 115: 179-185, 2005.

49. Voth M, Rosenberg M and Breuer J: Safety of gadobutrol, a new generation of contrast agents: Experience from clinical trials and postmarketing surveillance. Invest Radiol 46: 663-671, 2011.

50. Lang DM, Alpern MB, Visintainer PF and Smith ST: Elevated risk of anaphylactoid reaction from radiographic contrast media is associated with both beta-blocker exposure and cardiovascular disorders. Arch Intern Med 153: 2033-2040, 1993.
51. Gonzalo-Garijo MA, de Argila D, Pimentel JJ, Alejo M and García-Menaya JM: Skin reaction to contrast medium. Allergy 52: 875-876, 1997.

52. Dillman JR, Ellis JH, Cohan RH, Strouse PJ and Jan SC: Frequency and severity of acute allergic-like reactions to gadolinium-containing i.v. contrast media in children and adults. AJR Am J Roentgenol 189: 1533-1538, 2007.

53. Hox V, Desai A, Bandara G, Gilfillan AM, Metcalfe DD and Olivera A: Estrogen increases the severity of anaphylaxis in female mice through enhanced endothelial nitric oxide synthase expression and nitric oxide production. J Allergy Clin Immunol 135: 729-736, 2015.

54. Sze G, Brant-Zawadzki M, Haughton VM, Maravilla KR, McNamara MT, Kumar AJ, Aisen AM, Dreisbach JN, Bradley WG Jr and Weinreb JC: Multicenter study of gadodiamide injection as a contrast agent in MR imaging of the brain and spine. Radiology 181: 693-699, 1991. International (CC BY-NC-ND 4.0) License. 